\title{
Fault Tolerance for Active Surface System with Actuator Faults
}

\author{
Danqing Zhang $\mathbb{D},{ }^{1,2}$ Binbin Xiang $\mathbb{D},{ }^{1}$ Aili Yusup, ${ }^{1}$ Na Wang, ${ }^{1}$ and Guljaina Kazezkhan $\mathbb{D}{ }^{1,2}$ \\ ${ }^{1}$ Xinjiang Astronomical Observatory, Chinese Academic of Sciences, Urumqi 830011, China \\ ${ }^{2}$ University of Chinese Academy of Sciences, Beijing 100049, China \\ Correspondence should be addressed to Binbin Xiang; xiangbinbin@xao.ac.cn
}

Received 8 December 2020; Revised 31 January 2021; Accepted 17 February 2021; Published 26 February 2021

Academic Editor: Sándor Frey

Copyright (c) 2021 Danqing Zhang et al. This is an open access article distributed under the Creative Commons Attribution License, which permits unrestricted use, distribution, and reproduction in any medium, provided the original work is properly cited.

\begin{abstract}
The QiTai Radio Telescope (QTT) will be equipped with the active surface adjustment system (ASAS) to correct the main reflector deformation caused by environmental loading. In order to guarantee the stability and performance of the active surface system under fault conditions, it is necessary to adopt the fault-tolerant method when actuator faults have occurred. In this paper, a fault control method based on actuator faults weighting is proposed to solve the active surface fault control problem. According to the coordinates of the adjustable points of the panels corresponding to the faulty actuators, a new paraboloid is fitted by a weighted health matrix, and the fitting surface is taken as the target to adjust the surface shape.
\end{abstract}

\section{Introduction}

The QiTai Radio Telescope (QTT) is a general-purpose, high-precision radio telescope with observing frequency covering $150 \mathrm{MHz} 115 \mathrm{GHz}$ and the shortest observation wavelength is $3 \mathrm{~mm}$. In order to ensure efficient observation at the wavelength of $3 \mathrm{~mm}$, the required precision of the main reflector is very high. The precision of a single panel of the main reflector is required to be less than $0.08 \mathrm{~mm} \mathrm{rms} \mathrm{(root-mean-square)} \mathrm{[1].} \mathrm{The} \mathrm{surface} \mathrm{de-}$ formation of the main reflector is required to be less than $0.2 \mathrm{~mm} \mathrm{rms}$ after long-term correction by the active surface adjustment system (ASAS) [2].

When the best-fit surface is used as the reference surface of the ASAS, the deformation of the main reflector can be decreased. Many scholars have done a lot of work on the design of the best-fit surface. Hua proposed an optimal best-fit surface design based on the least-squares method at first [3], but there was a problem that the fitting surface was not the optimal surface when the elevation angle of the main reflector is $45 \mathrm{deg}$. After a further study based on the study of Hua, Chen found that the algorithm with the axial error as the minimum fitting quantity is the best [4]. Leng used the original algorithm optimized, which emphasizes the axial error as the minimum fitting quantity and finally, a more reasonable adjustment amount can be given [5]. This algorithm was verified under two working conditions 0 and 90 deg elevation.

In order to correct the deformation of the main reflector caused by external factors such as gravity, temperature, and wind, the shape of the reflector surface is adjusted by the ASAS by controlling the adjustment amount of actuators located between the reflector and the steel supporting truss, so that the antenna can still maintain less deformation when it is elevating and rotating [6,7]. An actuator can support and control four adjacent panels for the ASAS. The redundancy of the adjustment points of a single panel is insufficient. Therefore, when some actuators fail, they cannot rely on their own backlash to compensate for the structural gravity deformation. At present, there is no related research about the design of best-fit surfaces with faulty actuators. Therefore, it is significant to study the fault tolerance method for the ASAS with faulty actuators. This fault-tolerant method can be used as a reference for observers. It is suitable for special occasions where the current observation arrangement cannot be paused or actuators to be repaired cannot be replaced in time.

\section{Failure Mechanism Analysis of Active Surface}

The ASAS of the telescope is mainly composed of a master computer, control network, control bus, and actuators $[8,9]$. 
As a high-precision telescope with a large number of complex machinery and sensors, QTT will have advanced fault detection technology and complex process. The common failure types, failure causes, failure phenomena, and characteristics of the ASAS are shown in Table 1.

The fault diagnosis of the ASAS mainly depends on the fault diagnosis system of the telescope control system. The fault diagnosis system needs a series of advanced and intelligent methods, for example, Expert system, Fault tree [13], Neural network, and Fuzzy system. The advantages, disadvantages, and limitations of the various diagnostic methods are shown in Table 2.

The ASAS for QTT will adopt a semiclosed loop control system to correct surface deformation by controlling more than 2000 actuators. The fault tree model is especially suitable for the fault diagnosis of such a highly complex mechanism. Figure 1 shows the fault tree that enables us to make qualitative and quantitative analyses of the ASAS of the telescope and to deduce the minimum cut set [14] to determine specific faults.

The reasoning process of the fault tree analysis is shown in Figure 2. The inference mechanism based on the fault tree begins with the framework structure of the top event of the fault tree in the knowledge base, then the production of the framework is found, the production with high probability reasoning is selected, and finally, the cause of the fault from top to bottom is located.

\section{Fault Analysis of Actuators for the ASAS}

Actuator faults are one of the most frequent malfunctions in the ASAS. Actuator faults will prevent the adjustable main reflector from reaching the designated position, and it will affect the gain of the radio telescope. The decline of antenna efficiency caused by actuator faults is irreversible. However, it is difficult to replace the actuators immediately. The maintenance and replacement of the actuators for the Tian Ma Telescope and the FAST is a big challenge $[17,18]$. The ASAS of QTT will contain more than 2000 actuator nodes [19]. Therefore, it is crucial for maintenance personnel to know fault location and fault type before maintenance.

3.1. The Classification of Actuator Faults States. The state of actuator faults can be generally divided into four categories:

(1) Failure of a single actuator in a single sector A condition in which a single node in an area of the telescope cannot be adjusted due to a mechanical or electrical failure of a single actuator.

(2) Failure of multiple actuators in a single sector

The condition is caused by the failure of multiple actuators or circuits in the same area, the field bus that controls the sector, and the failure or blockage of the junction box.

(3) Failure of a single actuator in multiple sectors

A condition in which multiple nodes cannot be adjusted due to faults in the mechanical structure or circuit of a single actuator distributed over multiple areas of the telescope.

(4) Failure of multiple actuators in multiple sectors

The condition caused by the failure of the mechanical structure or the circuits of multiple actuators in multiple areas, the field buses controlling these sectors, and the failure and blockage of junction boxes.

The failure forms of the active plane are analyzed by measurement system and fault diagnosis system. Finally, the specific model of telescope failure is obtained.

3.2. Analysis of Failure Modes of Multiple Actuators. The ASAS of QTT will adopt distributed control to achieve the purpose of parabolic shape preserving by controlling the displacement of the piston of each actuator. When the actuator faults, the corner of the panel supported by the actuator cannot reach the specified position or gets stuck completely. In order to describe the failure degree of actuators, the failure factor is defined by

$$
\zeta= \begin{cases}1, & \text { normal, } \\ 0 \sim 1, & \text { partial failure, } \\ 0, & \text { complete failure, }\end{cases}
$$

where $\zeta$ is close to 1 , it indicates that the actuators are less damaged; $\zeta$ close to 0 , it indicates that the actuators are highly damaged [20]. When an actuator is working normally, the failure factor is $\zeta=1$; when an actuator is completely unable to work, the failure factor is $\zeta=0$; when an actuator is a partial failure, it still has a certain execution ability, the failure factor is $0<\zeta<1$.

According to the failure factor defined in equation (1), and the actual capacity of actuators displacement under different fault degrees is defined by the following:

$$
x= \begin{cases}x_{0}, & \text { normal, } \\ \zeta x_{0}, & \text { partial failure, } \\ 0, & \text { complete failure, }\end{cases}
$$

where $x$ is the actual displacement of the actuator, $x_{0}$ is the displacement of the actuator given by the computer. When the failure factor is 1, it represents there is no fault for the actuator, the displacement of the actuator is the same as before; when the failure factor is 0 , it represents the actuator is a complete failure, the actual displacement of the actuator is 0 ; when the failure factor is $0<\zeta<1$, it represents there is a partial failure for the actuator, the actual displacement of the actuator is $\zeta x_{0}$.

\section{Active Surface Fault Diagnosis System}

Fault tolerance of the main reflector consists of three parts, ASAS, Active surface fault diagnosis, and measuring subsystem. Through data analysis and real-time detection, the fault diagnosis and fault-tolerant method of actuator faults are realized. After the failure occurs in the ASAS, the 
TABLE 1: QTT failure mechanism analysis at the ASAS [10-12].

\begin{tabular}{|c|c|c|c|c|}
\hline & Types & Reason & Phenomena & Characteristics \\
\hline $\begin{array}{l}\text { Master } \\
\text { computer }\end{array}$ & $\begin{array}{l}\text { Accidental termination of } \\
\text { program computer failure }\end{array}$ & $\begin{array}{l}\text { Computer failure and } \\
\text { program crashes }\end{array}$ & $\begin{array}{l}\text { The whole system stopped } \\
\text { working }\end{array}$ & $\begin{array}{l}\text { Sudden failure and } \\
\text { obvious fault } \\
\text { characteristics }\end{array}$ \\
\hline $\begin{array}{l}\text { Control } \\
\text { network }\end{array}$ & $\begin{array}{l}\text { Ethernet failure, CAN bus } \\
\text { fault, junction box failure }\end{array}$ & $\begin{array}{l}\text { Data interface layer and } \\
\text { user interface layer failure }\end{array}$ & $\begin{array}{l}\text { Uncontrollable phenomena } \\
\text { occurring in multiple sectors }\end{array}$ & $\begin{array}{l}\text { Sudden failure and } \\
\text { obvious fault } \\
\text { characteristics }\end{array}$ \\
\hline Control bus & $\begin{array}{l}\text { Interruption, information } \\
\text { redundancy }\end{array}$ & $\begin{array}{l}\text { The protocol and the lines } \\
\text { are not connected }\end{array}$ & $\begin{array}{c}\text { The issuing command actuators } \\
\text { does not accept }\end{array}$ & $\begin{array}{l}\text { Easy to observe the } \\
\text { failure features }\end{array}$ \\
\hline $\begin{array}{l}\text { Displacement } \\
\text { actuator }\end{array}$ & $\begin{array}{l}\text { Worm gear pair [12], ball } \\
\text { screw pair, power module, } \\
\text { stepper motor, limit switch } \\
\text { failure }\end{array}$ & $\begin{array}{l}\text { Stuck, line aging, } \\
\text { mechanical equipment } \\
\text { elastic deformation or } \\
\text { gluing, output deviation }\end{array}$ & $\begin{array}{l}\text { The actuator fails to reach the } \\
\text { specified pointing coordinate } \\
\text { position and sends out abnormal } \\
\text { sound }\end{array}$ & $\begin{array}{l}\text { Sudden failure, obvious } \\
\text { fault features, features } \\
\text { difficult to extract }\end{array}$ \\
\hline
\end{tabular}

TABLE 2: Active surface fault diagnosis method based on artificial intelligence [13-15].

\begin{tabular}{|c|c|c|}
\hline Method & Advantage & Limitation \\
\hline Expert system & Strict reasoning logic, high reliability & Knowledge is difficult to obtain, complex reasoning \\
\hline Fault tree & $\begin{array}{c}\text { Qualitative, quantitative analysis with logical } \\
\text { reasoning }\end{array}$ & $\begin{array}{c}\text { The construction process is heavy and difficult. Logical operations are } \\
\text { prone to error }\end{array}$ \\
\hline $\begin{array}{l}\text { Neural } \\
\text { network }\end{array}$ & $\begin{array}{l}\text { Parallel processing, self-learning, self- } \\
\text { organization, }\end{array}$ & The reasoning process of diagnosis is not clear \\
\hline $\begin{array}{l}\text { Fuzzy } \\
\text { inference }\end{array}$ & $\begin{array}{c}\text { Flexible enough to deal with uncertain } \\
\text { information }\end{array}$ & It is difficult to establish rules and membership functions \\
\hline
\end{tabular}

relevant information in the log system is transmitted into the active surface fault diagnosis system, which is selected by the discriminant module for fault-tolerant processing and triggered by the alarm system to report the current active surface status information to the engineer. Fault-tolerant processing is carried out for actuator faults. The results are updated and stored in the log system, and the adjustment amount is fed back to the ASAS. The master computer controls the displacement actuators to reach the new position value. The scenario is completed by the cooperation of the telescope fault diagnosis system, the ASAS, and measuring system. Active surface fault diagnosis system is based on the development of fault handling system of large telescope control system [21]. The fault diagnosis process is shown in Figure 3.

The mechanical stress and stiffness of the panels and actuators are considered in the active surface fault diagnosis system. Thus, the best-fit paraboloid of health matrix combined weighting based on this information can effectively improve the surface shape precision after the fault.

\section{Optimal Weighted Best-Fit Paraboloid}

When actuators are a failure, the main reflector model has heteroscedasticity, and the weighted best-fit is used to solve the fitting parameters of the model so that it does not follow the heteroscedasticity. Based on the idea of traditional bestfit surface design [3], the optimal weighted fit method adds the concept of weighting and focuses on considering the fault condition of actuators. Based on the method, when there is a fault for the small number of actuators, in order to ensure operating efficiency, the telescope and the ASAS will not be stopped and maintained immediately, but keep on working with fault tolerance controlling of the ASAS by the optimal weighted best-fit method.

\subsection{The Traditional Method for Calculating the Best-Fit} Surface. The most general method of the best-fit is the leastsquares method, which minimizes the deformation from more than 2,000 actuator errors.

$$
Q=\sum_{i=1}^{P} \delta_{i}^{2},
$$

where $\delta_{i}$ is the error of the $i$ th actuator residual value, $Q$ is the sum of the squares of the residual errors of the entire main reflector, $p$ is the number of actuators.

The shape of the main reflector surface can be obtained by reflector surface measurement, so surface deformation comes from the same distribution. Statistically, the leastsquare estimation parameter is the maximum likelihood estimation method whose error distribution is Gaussian noise.

QTT will adopt a Gregorian antenna, and its main reflector is a paraboloid and subreflector is an ellipsoid. Suppose the equation of the original designed paraboloid $S$ is as follows:

$$
z=\frac{x^{2}+y^{2}}{4 f}
$$

where $f$ is the focal length of paraboloid, $x, y, z$ is theoretical coordinates value, and $x_{i}, y_{i}, z_{i}$, is the theoretical position of the $i$ th actuator.

The actual surface $S_{1}$ consists of rigid body displacement and elastic deformation relative to the design surface, and 


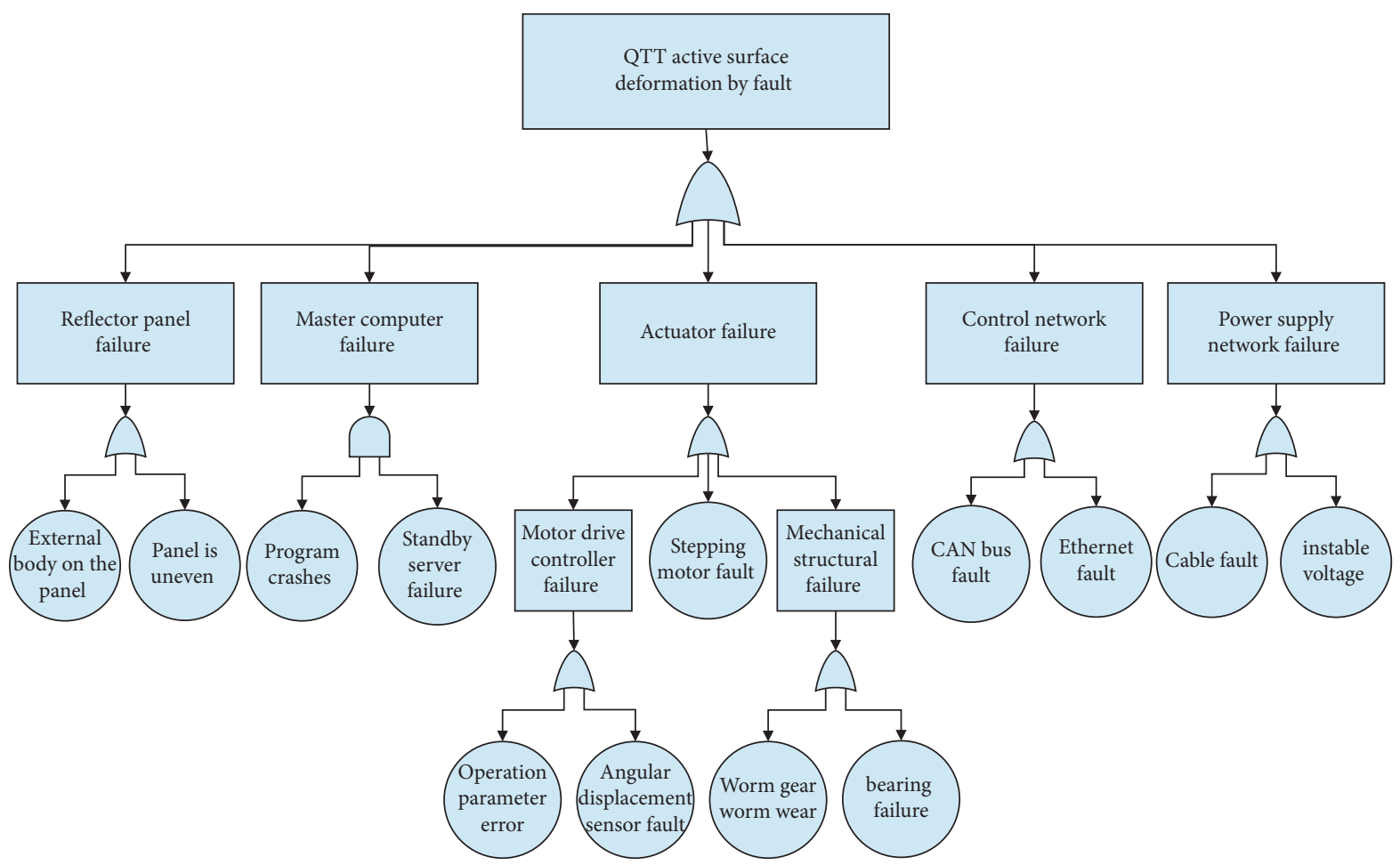

FIgURE 1: Active surface fault tree analysis [15].

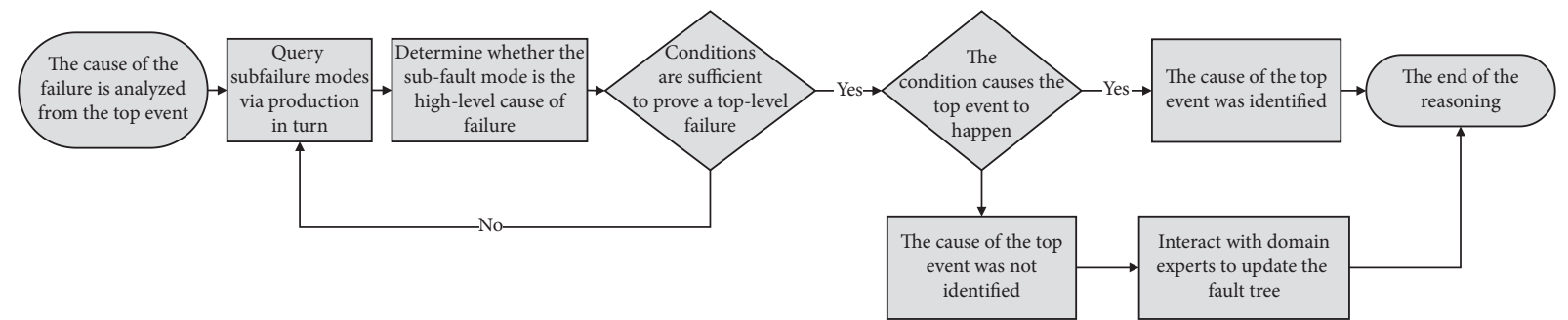

FIGURE 2: Reasoning process of the fault tree analysis [16].

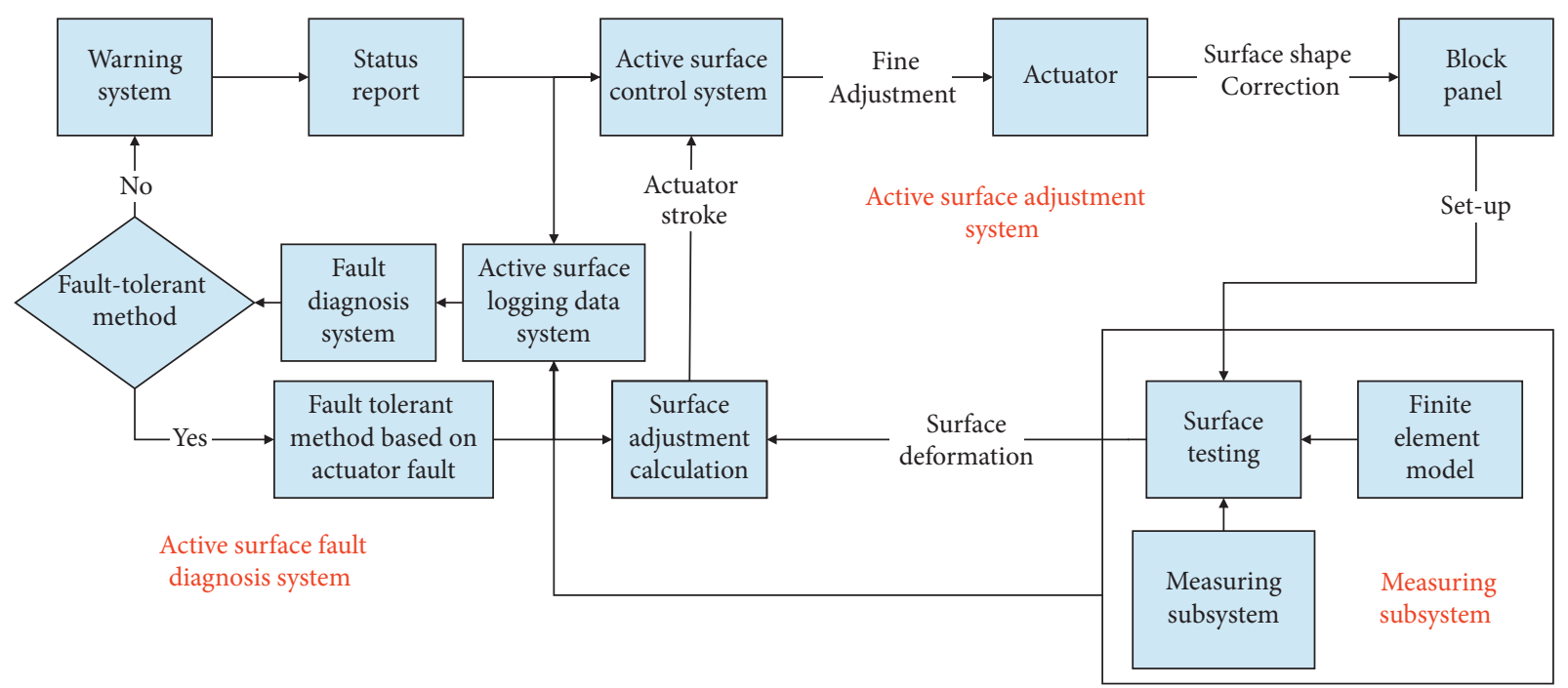

Figure 3: Active surface fault diagnosis flow chart. 
there are 6 variates of coordinates in the best-fit surface relative to the originally designed coordinates: $\Delta x, \Delta y, \Delta z, \Delta \varphi, \Delta \psi, \Delta f$. These parameters are called by fitting parameters in fault condition. When the 6 fitting parameters are known, the best-fit surface is determined and it has minimum rms deformation.

The axial coordinate $z_{i}^{\prime}$ of any point on the design surface $S_{0}$ on the best-fit surface $S_{2}$ is approximately

$$
z_{i}^{\prime} \approx \frac{\left(x_{i}-\Delta x\right)^{2}+\left(y_{i}-\Delta y\right)^{2}}{4(f+\Delta f)}+y_{i} \Delta \varphi-x_{i} \Delta \psi+\Delta z .
$$

$$
\begin{aligned}
& \text { Simplify } \\
& z_{i}^{\prime}=z_{i}-\frac{x_{i}}{2 f} \Delta x-\frac{y_{i}}{2 f} \Delta y-\frac{z_{i}}{f} \Delta f-x_{i} \Delta \psi+y_{i} \Delta \varphi+\Delta z .
\end{aligned}
$$

Then, the least-squares matrix form of axial error between the measured value $z_{i}^{*}$ and the best-fit coordinate values $z_{i}^{\prime}$ at any point are as follows:

$$
\left\{\begin{array}{cccccc}
-\frac{x_{1}}{2 f} & -\frac{y_{1}}{2 f} & 1 & y_{1} & -x_{1} & -\frac{z_{1}}{f} \\
-\frac{x_{2}}{2 f} & -\frac{y_{2}}{2 f} & 1 & y_{2} & -x_{2} & -\frac{z_{2}}{f} \\
\cdots & \cdots & \cdots & \cdots & \cdots & \cdots \\
-\frac{x_{p}}{2 f} & -\frac{y_{p}}{2 f} & 1 & y_{p} & -x_{p} & -\frac{z_{p}}{f}
\end{array}\right\}\left\{\begin{array}{c}
\Delta x \\
\Delta y \\
\Delta z \\
\Delta \varphi \\
\Delta \psi \\
\Delta f
\end{array}\right\}=\left\{\begin{array}{c}
z_{1}^{*}-z_{1}^{\prime} \\
z_{2}^{*}-z_{2}^{\prime} \\
\cdots \\
z_{p}^{*}-z_{p}^{\prime}
\end{array}\right\}
$$

Abbreviation:

$$
[\mathbf{C}][\mathbf{D}]=[\mathbf{H}] .
$$

Regularization:

$$
[\mathbf{C}]^{\mathrm{T}}[\mathbf{C}][\mathbf{D}]=[\mathbf{C}]^{\mathrm{T}}[\mathbf{H}] .
$$

where $D=\{\Delta x, \Delta y, \Delta z, \Delta \varphi, \Delta \psi, \Delta f\}^{T}$ is fitting parameters. The fitting parameters can be obtained by the least-squares method [3], and the calculation of best-fitting of the paraboloid is deduced by weighted optimization in subsequent sections.

\subsection{The Calculation Method of Optimal Weighted Best-Fit} Paraboloid. Firstly, the concept of a health matrix is introduced to describe the performance status of each actuator. The health matrix is used as a penalty term in the calculation of the best-fit paraboloid based on the weighted surface to constrain faulty actuators displacement so as to ensure that these actuators can match optimal weighted best-fit paraboloid surface without moving, and decrease the rms surface error and consequently the antenna gain is improved under the condition of fault.

The heath matrix is given by the active surface fault diagnosis system according to the failure factors of each actuator. The health state of actuators is represented by the value of 0,1 and reciprocal of the failure factor of actuators, 0 represents complete failure, 1 represents health, and other values represent partial failure. The larger the value, the higher the failure degree. The health matrix is expressed and the transformation parameters are solved by combining the equation group of the parameter matrix. The best-fit paraboloid based on optimal weighted will pay more attention to the information of the fault point so that the transformation of the actual fault paraboloid to the optimal weighted best-fit paraboloid will take the invariance of the fault point as the design benchmark to achieve a faulttolerant effect. The penalty function of the fault point can be defined as follows:

$$
\delta=\sum_{i=1}^{p} a_{i}\left(z_{i}^{*}-z_{i}^{\prime}\right)^{2},
$$

where $a_{i}$ is the constant with noncanonical constraint, $\delta$ is the surface error of the whole main reflector surface. This will bring the target parameter closer to the optimal value. The matrix form is used to solve the fitting parameters of the best-fit paraboloid based on optimal weighted.

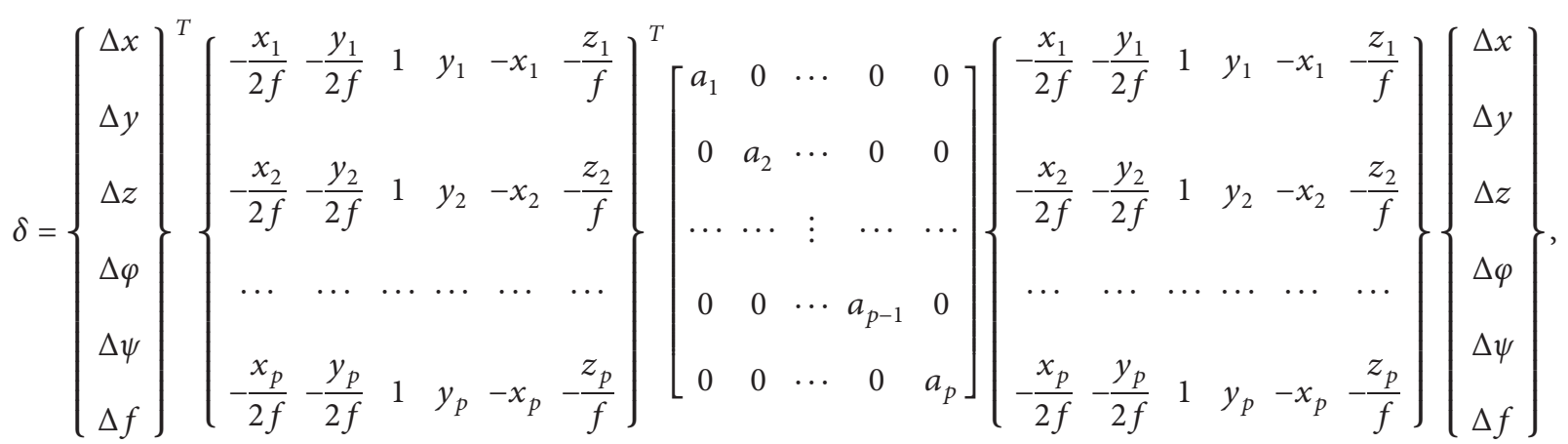

where $\mathrm{W}$ represents the health matrix of the faulty surface.

The theoretical design of $\mathrm{W}$ needs to consider the fault tree model of the active surface fault diagnosis system to get actuators reliability and then to get the malfunction probability of the Top event. In order to facilitate the simulation, a general and concise expression is given in this paper. 
The actuator's health degree can be defined by the reciprocal of the failure factor for each actuator. A definition of failure factor is given by the following equation:

$$
\zeta_{i}=1-\frac{x_{i}^{*}-x_{i}^{\prime}}{x_{i}^{*}}+\text { eps, }
$$

where $x_{i}^{*}$ is the displacement given by the master control computer, and $x_{i}^{\prime}$ is the actual displacement, epsrepresents the relative precision of floating point, which is to avoid the situation the denominator is zero. The health matrix $\mathbf{W}$ can be given by the following:

$$
\mathbf{W}=\left[\begin{array}{ccccc}
\zeta_{1}^{-1} & 0 & \cdots & 0 & 0 \\
0 & \zeta_{2}^{-1} & \cdots & 0 & 0 \\
\cdots & \cdots & \vdots & \cdots & \cdots \\
0 & 0 & \cdots & \zeta_{P-1}^{-1} & 0 \\
0 & 0 & \cdots & 0 & \zeta_{P}^{-1}
\end{array}\right] .
$$

The expression of $D=\{\Delta x, \Delta y, \Delta z, \Delta \varphi, \Delta \psi, \Delta f\}^{T}$ gradient $\nabla \delta$ is as follows:

$$
\begin{aligned}
& \nabla \delta=\frac{\partial \delta}{\partial \mathbf{D}}=\mathbf{C}^{\mathrm{T}} \mathbf{W} \mathbf{C D}+\mathbf{C}^{\mathrm{T}} \mathbf{W}^{\mathrm{T}} \mathbf{C D}=\mathbf{C}^{\mathrm{T}}\left(\mathbf{W}^{\mathrm{T}}+\mathbf{W}\right) \mathbf{C D} \\
& \mathbf{D}_{n}=\mathbf{D}_{n-1}-\alpha \nabla \delta
\end{aligned}
$$

An initial value $D_{0}$ is used to solve an optimal solution of $D$ by gradient descent (GD), and $\alpha$ is the optimal step size.

\section{Simulation Experiment of Fault Tolerance Method with Actuator Faults}

It is assumed that a 110-meter telescope has a focal diameter ratio of 0.33 . The entire ASAS is divided into 32 sectors, and 64 actuators are distributed in each sector. For the convenience of data simulation, an actuator is assumed to control four adjacent panels except for the center and boundary of the paraboloid, totaling 2048 actuators. The fault residual after actuator faults is expressed as a random distribution. By simulating and comparing the stroke of fault points position and nonfault points position, the best-fit surface is designed under the fault points, which are not moved as much as possible.

The positioning error of the actuators is a group of Gaussian noises subject to small variance, and the SNR of surface measurement is set to $20 \mathrm{~dB}$. Different colors represent the random distribution of errors. Error distribution of the actual error of the fault paraboloid is shown in Figure 4. The deeper blue areas represent the degree and location of the fault. The position in the white circle represents the specific fault location.

Combining fitting parameters and coordinate transformation formula, a new ideal coordinate of the paraboloid surface was obtained [22].

$$
\left\{\begin{array}{l}
x_{n}=\left(x_{0}-\Delta x\right)-z_{0} \Delta \psi \\
y_{n}=\left(y_{0}-\Delta y\right)+z_{0} \Delta \varphi \\
z_{n}=\left(z_{0}-\Delta z\right)+x_{0} \Delta \psi+y_{0} \Delta \varphi \\
f_{n}=f_{0}+\Delta f
\end{array}\right.
$$

Compared with the surface error after surface corrected when actuators worked normally, actuators were partial fault, and the ASAS adopted fault-tolerant method after the fault occurred at 0,20,35, and 70 deg elevation, respectively. The results are shown in Table 3 .

We assume that the main reflector rms surface error after the ASAS enabled is 0 under ideal conditions, which is not to consider the influence of the positioning precision of the actuators. Selecting 15 actuators as actual fault actuators randomly, perform simulation and take the fault tolerance method for the main reflector with faulty actuators. Comparing the surface error with faulty actuators between fault-tolerant method enabled and disabled, it is clear that rms error is far from half at 0 deg elevation, reduced to about half at 20, $35 \mathrm{deg}$ elevation, and also reduced around $0.06 \mathrm{~mm}$ rms at $70 \mathrm{deg}$ elevation.

Due to the excessive number of actuators, 400 actuators were randomly selected for simulation analysis and comparison. The surface deformation and position of the piston of actuators with the nonfault and at different elevations are shown in Figures 5-12. It is obvious that the displacement range of the actuator with nonfault is smaller than that with the fault-tolerant method. The red line chart and blue line chart indicate piston position of no-faulty actuators before and after adopting the faulttolerant method, respectively. It is obvious that the fluctuation of the red line chart range is relatively greater than the blue line chart range. The piston position range changes about 1-4 mm compared with the unadopted fault-tolerant method.

Figures 6, 8, 10, and 12 represent the main reflector deformation distribution at $0,20,35$, and $70 \mathrm{deg}$ elevation, (a) represents the main reflector deformation when the ASAS disabled, (b) represents the main reflector deformation including failure of actuators when the ASAS enabled, (c) represents the main reflector deformation include failure of actuators after adopting the fault tolerance method. Blue dots represent the location of the failure of actuators. After taking optimal weighted best-fit paraboloid as the reference surface, most of the fault point deformation is reduced. Finally, the goal of reducing the main reflector $\mathrm{rms}$ is achieved.

In other words, in the case of complete or partial failure of the fault point, the redundancy adjustment ability of the nonfault point can make up for the lack of surface precision and improve the antenna gain and observation efficiency of the telescope. The availability of this method can be verified by these examples. We think the method can be used as an effective fault-tolerant method for telescope maintenance management. 


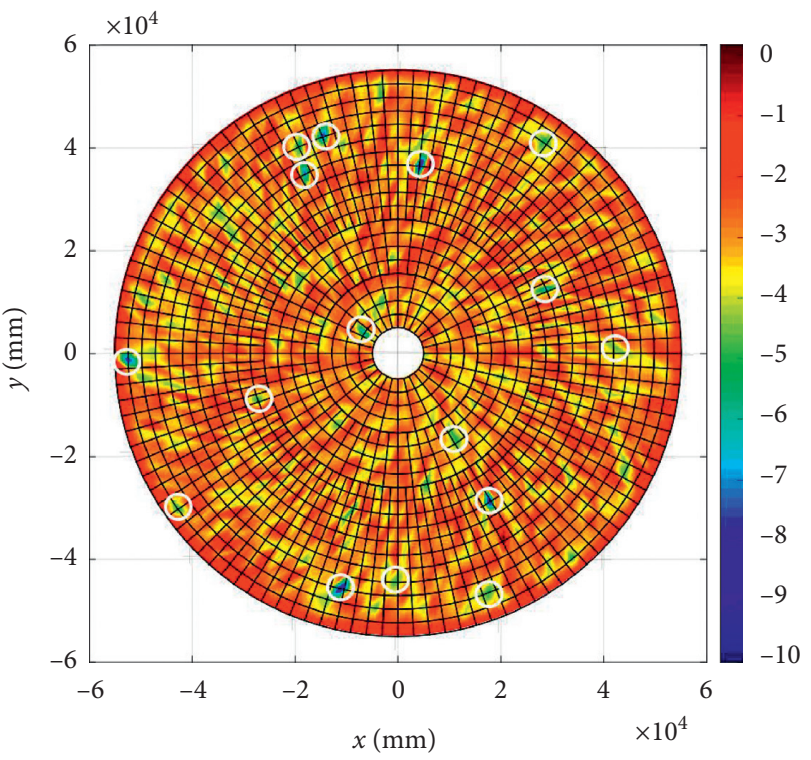

FIGURE 4: Main reflector in case of actuator failure.

TABLE 3: Comparison of the main reflector rms after adopting fault-tolerant method.

\begin{tabular}{|c|c|c|c|c|}
\hline Elevation angle (deg) & 0 & 20 & 35 & 70 \\
\hline Surface error when ASAS disabled (mm rms) & 0.5300 & 0.3797 & 0.3300 & 0.4355 \\
\hline Surface error with no-faulty actuators (ideal) when ASAS enabled ( $\mathrm{mm} \mathrm{rms)}$ & 0 & 0 & 0 & 0 \\
\hline Surface error with faulty actuators when ASAS enabled ( $\mathrm{mm} \mathrm{rms)}$ & 0.2588 & 0.2819 & 0.2178 & 0.2834 \\
\hline Surface error with fault-tolerant when ASAS enabled by proposed method ( $\mathrm{mm} \mathrm{rms)}$ & 0.0701 & 0.1552 & 0.1332 & 0.2237 \\
\hline
\end{tabular}

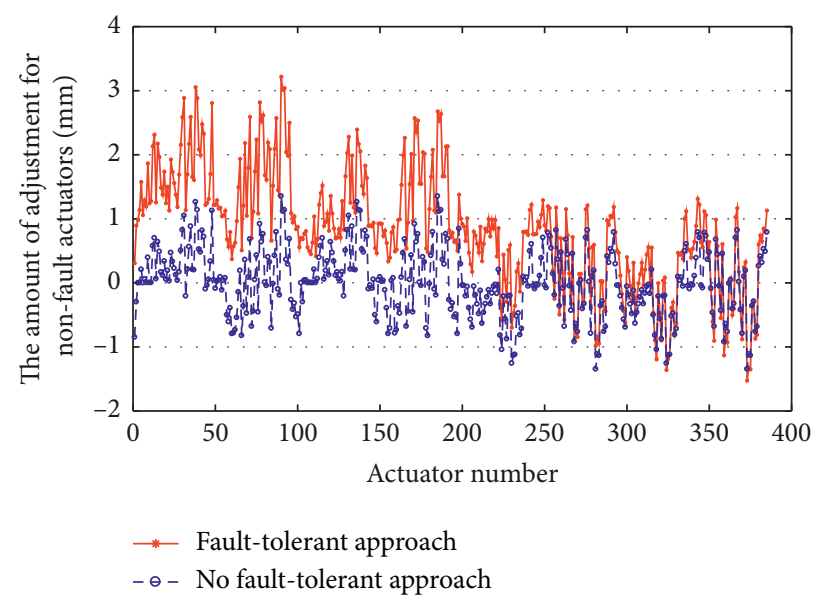

FIgURE 5: Nonfault actuators' stroke at 0 deg elevation. 


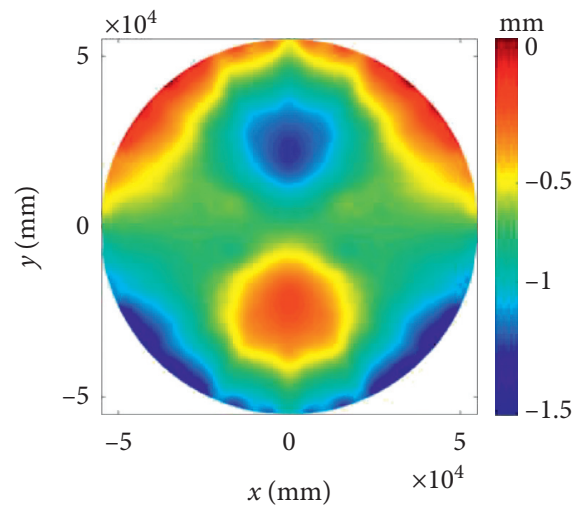

(a)

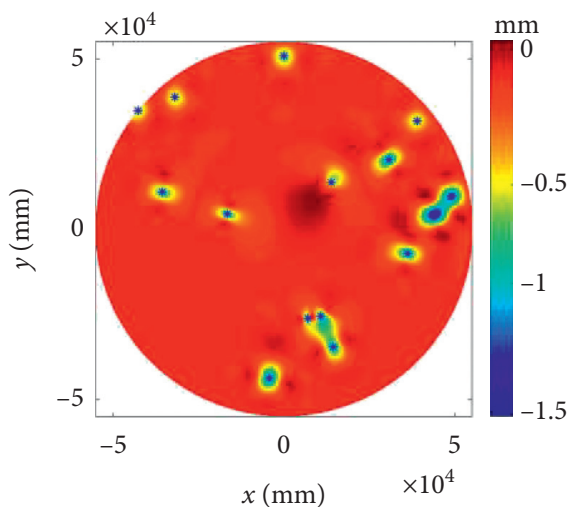

(b)

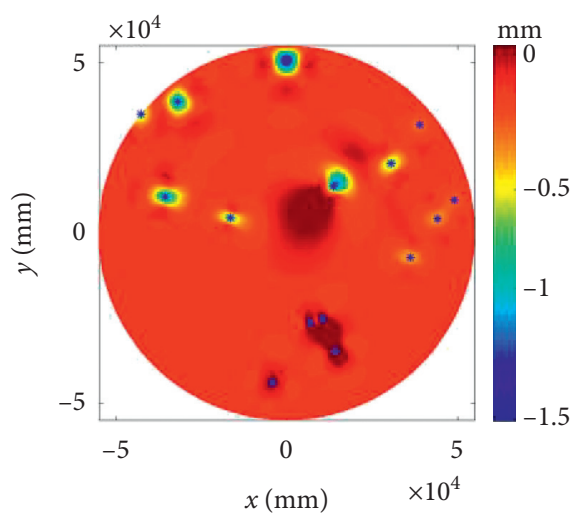

(c)

FIgURE 6: Compare with the main reflector rms deformation at 0 deg elevation. (a) The reflector deformation before actuator failure. (b) The reflector deformation after actuator failure. (c) The reflector deformation at tolerance method.

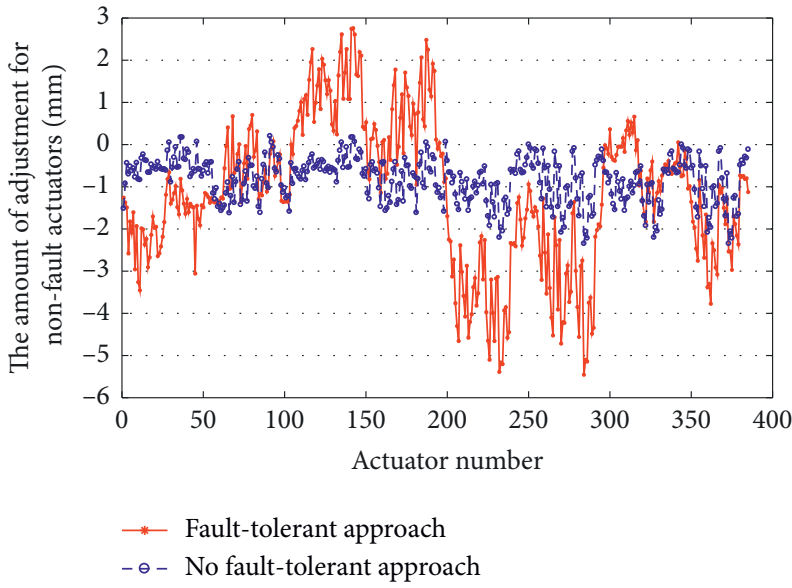

FIgURE 7: Nonfault actuators' stroke at 20 deg elevation. 


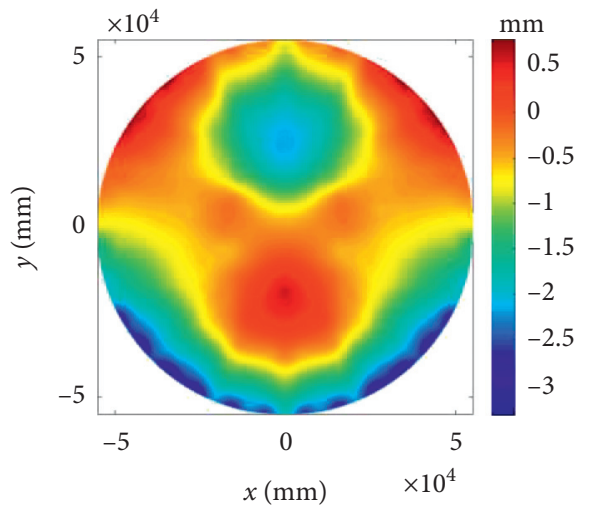

(a)

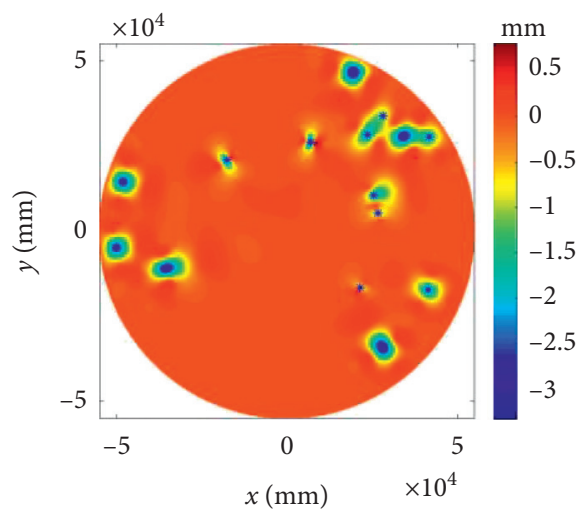

(b)

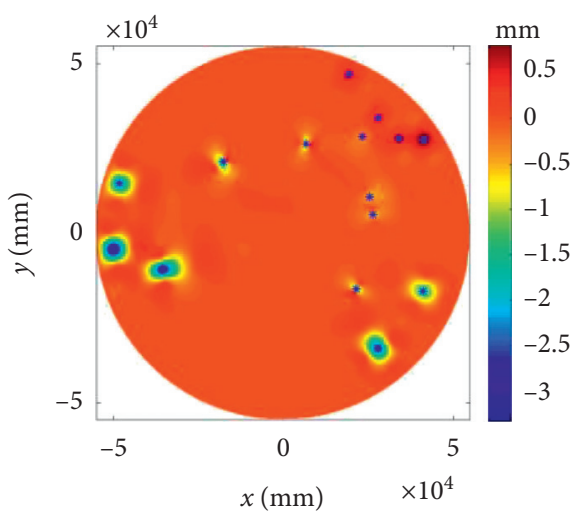

(c)

Figure 8: Compare with the main reflector deformation at 20 deg elevation. (a) The reflector deformation before actuator failure. (b) The reflector deformation after actuator failure. (c) The reflector deformation at tolerance method.

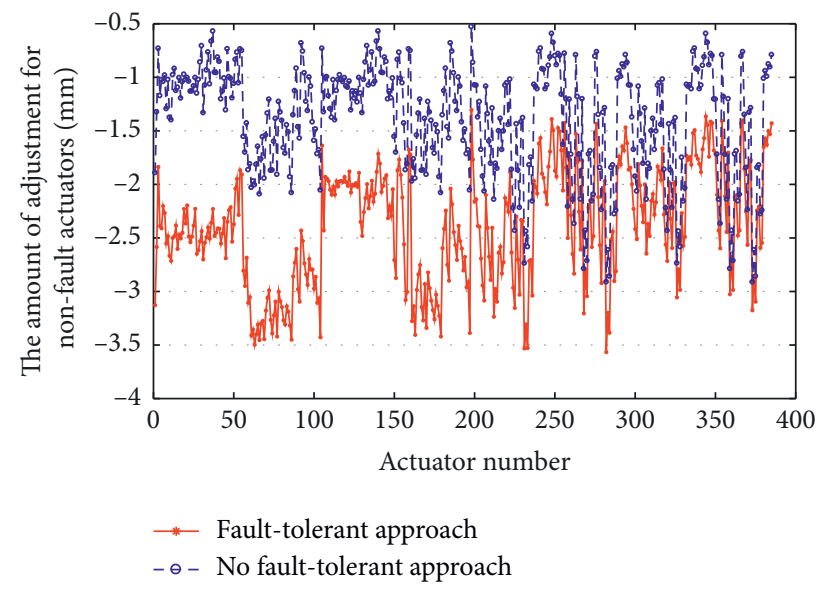

FIgURE 9: Nonfault actuators' stroke at $35 \mathrm{deg}$ elevation. 


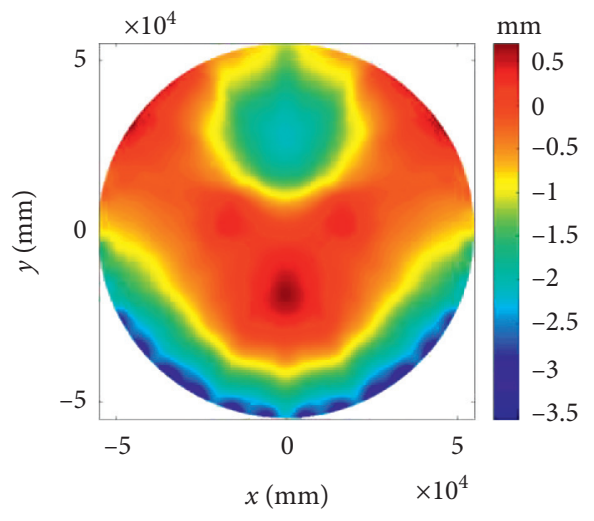

(a)

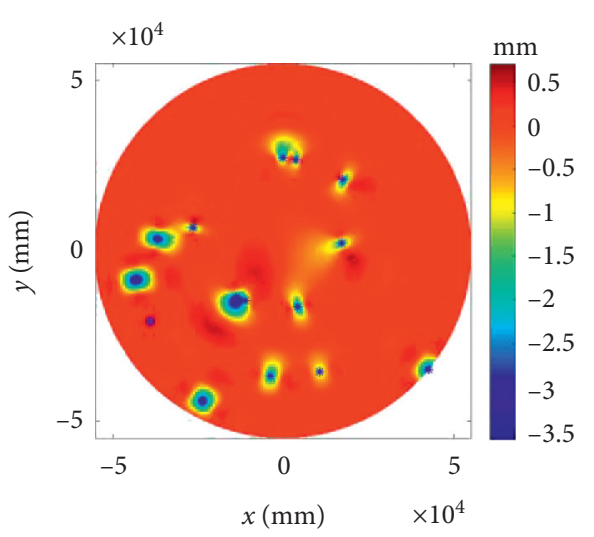

(b)

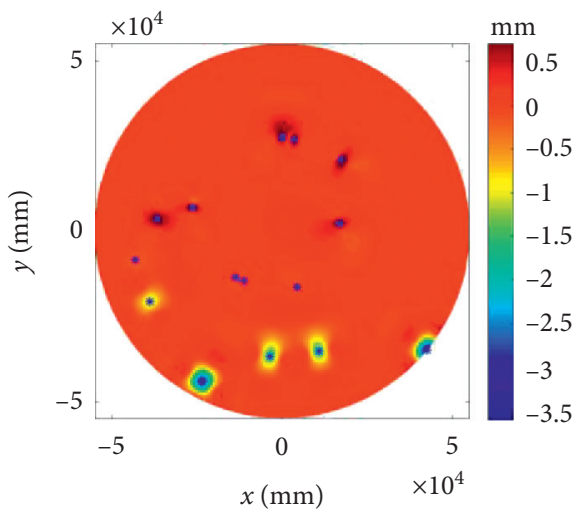

(c)

Figure 10: Compare with the main reflector deformation at 35 deg elevation. (a) The reflector deformation before actuator failure. (b) The reflector deformation after actuator failure. (c) The reflector deformation at tolerance method.

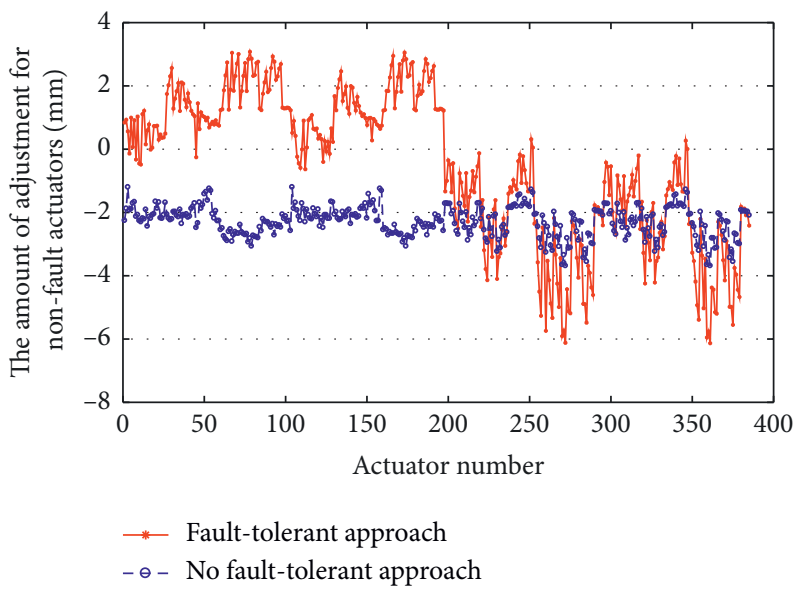

Figure 11: Nonfault actuators' stroke at 70 deg elevation. 


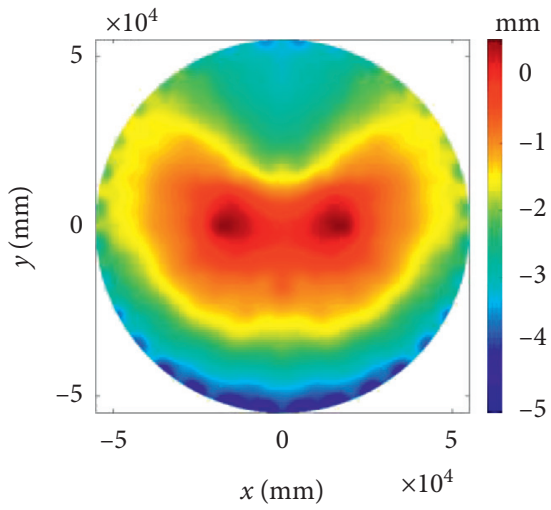

(a)

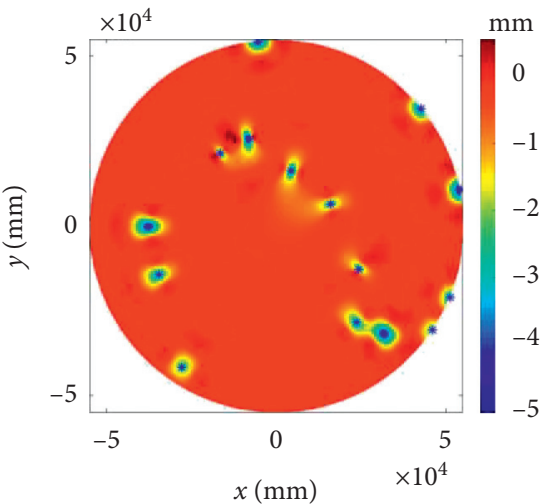

(b)

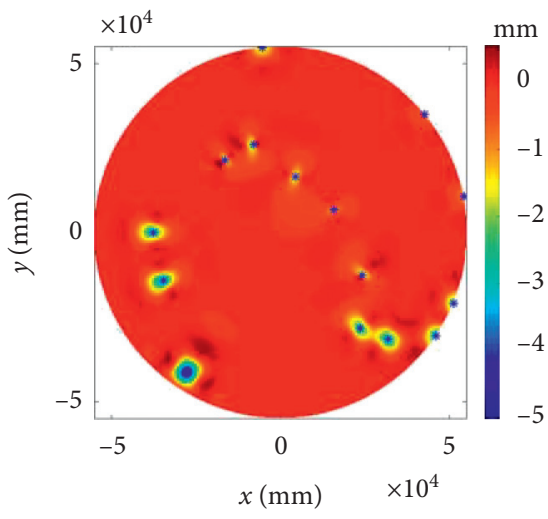

(c)

Figure 12: Compare with the main reflector deformation at 70 deg elevation. (a) The reflector deformation before actuator failure. (b) The reflector deformation after actuator failure. (c) The reflector deformation at tolerance method.

\section{Conclusion}

In summary, the fault tolerance method, which reduces the main reflector deformation under the situation of actuator faults, has been presented. This method keeps faulty actuators immobile or partially movable. First, the health matrix of actuators is used to fit optimal weighted best-fit paraboloid, and then these nonfaulty actuators are driven to compensate the main reflector deformation. Finally, the aim of improving the accuracy of the main reflector under the fault conditions is achieved. The scheme provides a theoretical basis and a strategic scheme for the observers to deal with malfunction.

\section{Data Availability}

No data were used to support this study.

\section{Conflicts of Interest}

The authors declare that they have no conflicts of interest.

\section{Acknowledgments}

This work was funded by the National Key Basic Research and Development Program (grant no. 2018YFA0404702) and the Chinese Academy of Sciences (CAS) "Light of West China" Program (grant no. 2017-XBQNXZ-B-021).This work was also partly supported by the Operation, Maintenance, and Upgrading Fund for Astronomical Telescopes and Facility Instruments, budgeted from the Ministry of Finance of China (MOF) and administrated by CAS. Also, the authors acknowledge the academy's technical support.

\section{References}

[1] N. Wang, "Xinjiang Qitai $110 \mathrm{~m}$ radio telescope," Scientia Sinica Physica, Mechanica \& Astronomica, vol. 44, no. 8, pp. 783-794, 2014, in Chinese.

[2] A. Orfei, M. Morsiani, G. Zacchiroli et al., "The active surface system on the noto radio telescope," in Proceedings on VLBI
Technology Progress and Future Observational Possibilities, Tokyo, Japan, September 2002.

[3] M. L. Hua, "A best-fit method for deformed surface of antennas," Modern Radar, vol. 1, pp. 75-82, 1994, in Chinese.

[4] J. H. Chen and G. Y. Li, "Research on discrete point parabolic fitting algorithm," Radio Engineering, vol. 35, no. 4, pp. 32-34, 2005, in Chinese.

[5] G. J. Leng, "Precise algorithm for best fit surface axis errors of reflector antenna," Chinese Journal of Radio Science, vol. 24, no. 5, pp. 826-831, 2009, in Chinese.

[6] C. S. Wang, H. H. Li, K. Ying et al., "Active surface compensation for large radio telescope antennas," International Journal of Antennas and Propagation, vol. 390, no. 3412, pp. 1687-5869, 2018, in Chinese.

[7] J. R. Lacasse, "Green bank telescope active surface system," Astronomical Telescopes and Instrumentation, vol. 33, no. 51, pp. 310-319, 1998.

[8] A. Orfel, M. Morsiani, G. Zacchiroli et al., "An active surface for large reflector antennas," IEEE Antennas \& Propagation Magazine, vol. 46, no. 4, pp. 11-19, 2004.

[9] G. Zacchiroli, A. Orfei, M. Morsiani et al., "A prototype of a mechanical actuator for active surface used on parabolic antennas," Istituto di Radioastronomia Technical Report IRA, vol. 20795 pages.

[10] Q. Feng, "Analysis of radar fault detection and diagnosis technology and new development," Electronic Component and Information Technology, vol. 08, pp. 18-23, 2018, in Chinese.

[11] D. Linaric and V. Koroman, "Fault diagnosis of a hydraulic actuator using neural network," in Proceedings of the IEEE International Conference on Industrial Technology, pp. 108111, IEEE, Maribor, Slovenia, May 2003.

[12] X. F. Jing, F. Fan, H. L. Qian et al., "Fault diagnosis of cablenet structure supporting active reflector of large radio telescope-FAST," China Civil Engineering Journal, vol. 43, no. 06, pp. 18-25, 2010, in Chinese.

[13] D. Li, "Fault diagnosis for a radar based on fault tree analysis and fuzzy theory," Computer Measurement \& Control, vol. 04, no. 28, pp. 496-497, 2007, in Chinese.

[14] B. O. Gaddouna and M. Ouladsine, "Fault diagnosis in a hydraulic process using unknown input observers," in Proceedings of the IEEE International Conference on Control Applications, pp. 108-111, IEEE, Hartford, CT, USA, August 1997. 
[15] R. Landau, Intelligent fault diagnosis at the Australia Telescope: Publications of the Astronomical Society of Australia, Cambridge University Press, Cambridge, UK, 1994.

[16] M. C. Luo, Design of Fault Diagnosis System for Central Control System of Large Telescope, University of Science and Technology of China, Hefei, China, 2014.

[17] J. X. Xue, Z. Lei, X. D. Gu et al., "A study of a design of a mechanism for rapidly dismounting and assembling a faulty FAST actuator," Astronomical Research \& Technology, vol. 12, no. 1, pp. 102-108, 2010, in Chinese.

[18] J. Dong, H. L. Jin, Q. Ye et al., "The active surface control system for the tian ma telescope," Astronomical Telescopes and Instrumentation, 2016.

[19] B. B. Xiang, F. Xue, N. wang et al., "Preliminary design of QTT active surface system control network," Electro-Mechanical Engineering, vol. 34, no. 6, pp. 51-56, 2018, in Chinese.

[20] B. Michal, "Diagnosing multiple faults with the dynamic binary matrix," IFAC-PapersOnLine, vol. 48, no. 21, pp. 1297-1302, 2015.

[21] J. Wang, M.-C. Luo, W.-Q. Wu et al., "Study of central control system for FAST," Experimental Astronomy, vol. 36, no. 3, pp. 569-589, 2013, in Chinese.

[22] Z. Ma, D. H. Yang, S. Q. Wang et al., "Antenna reflector surface fitting algorithm based on rigid body displacement principle," Journal of Mechanical Engineering, vol. 46, no. 18, pp. 29-35, 2010, in Chinese. 